\title{
Multi-Day Prolonged Low- to Moderate-Intensity Endurance Exercise Mimics Training Improvements in Metabolic and Oxidative Profiles Without Concurrent Chromosomal Changes in Healthy Adults
}

Dominique D. Gagnon ${ }^{1,2 *}$, Sandra Dorman ${ }^{1,2,3}$, Stephen Ritchie ${ }^{1,2}$, Shivaprakash Jagalur Mutt ${ }^{4,5}$, Ville Stenbäck $k^{4,5}$, Jarosław Walkowiak ${ }^{6}$ and Karl-Heinz Herzig 4,5,6

OPEN ACCESS

Edited by:

Hassane Zouhal,

University of Rennes 2 - Upper

Brittany, France

Reviewed by:

Issy Laher.

The University of British Columbia

Canada

Yi Sub Kwak,

Dong-eui University, South Korea

*Correspondence:

Dominique D. Gagnon ddgagnon@laurentian.ca; dodgagnon@gmail.com

Specialty section: This article was submitted to

Exercise Physiology,

a section of the journal

Frontiers in Physiology

Received: 13 December 2018

Accepted: 14 August 2019

Published: 03 September 2019

Citation:

Gagnon DD, Dorman S, Ritchie S, Mutt SJ, Stenbäck V, Walkowiak J

and Herzig K-H (2019) Multi-Day

Prolonged Low- to Moderate-Intensity Endurance Exercise Mimics Training

Improvements in Metabolic

and Oxidative Profiles Without Concurrent Chromosomal Changes in Healthy Adults.

Front. Physiol. 10:1123. doi: 10.3389/fphys.2019.01123
'Laboratory of Environmental Exercise Physiology, School of Human Kinetics, Laurentian University, Sudbury, ON, Canada, ${ }^{2}$ Center of Research in Occupational Safety and Health, Laurentian University, Sudbury, ON, Canada, ${ }^{3}$ Northern Ontario School of Medicine, Sudbury, ON, Canada, ${ }^{4}$ Research Unit of Biomedicine, Department of Physiology and Biocenter of Oulu, University of Oulu, Oulu, Finland, ${ }^{5}$ Medical Research Center, Oulu University Hospital and University of Oulu, Oulu, Finland, ${ }^{6}$ Department of Gastroenterology and Metabolism, Poznan University of Medical Sciences, Poznań, Poland

Background: Oxidative stress results in lipid, protein, and DNA oxidation, resulting in telomere erosion, chromosomal damage, and accelerated cellular aging. Training promotes healthy metabolic and oxidative profiles whereas the effects of multi-day, prolonged, and continuous exercise are unknown. This study investigated the effects of multi-day prolonged exercise on metabolic and oxidative stress as well as telomere integrity in healthy adults.

Methods: Fifteen participants performed a 14-day, 260-km, wilderness canoeing expedition (12 males) (EXP) (24 \pm 7 years, $72 \pm 6 \mathrm{~kg}, 178 \pm 8.0 \mathrm{~cm}, 18.4 \pm 8.4 \%$ BF, $\left.47.5 \pm 9.3 \mathrm{mlO}_{2} \mathrm{~kg}^{-1} \mathrm{~min}^{-1}\right)$, requiring $6-9 \mathrm{~h}$ of low- to moderate-intensity exercise daily. Ten controls participated locally (seven males) (CON) (31 \pm 11 years, $72 \pm 15 \mathrm{~kg}$, $174 \pm 10 \mathrm{~cm}, 22.8 \pm 10.0 \% \mathrm{BF}, 47.1 \pm 9.0 \mathrm{mlO}_{2} \mathrm{~kg}^{-1} \mathrm{~min}^{-1}$ ). Blood plasma, serum, and mononuclear cells were sampled before and after the expedition to assess hormonal, metabolic, and oxidative changes.

Results: Serum cholesterol, high- and low-density lipoprotein, testosterone, insulin, sodium, potassium, urea, and chloride concentrations were not different between groups, whereas triglycerides, glucose, and creatinine levels were lower following the expedition $(p<0.001)$. Malondialdehyde and relative telomere length $(T L)$ were unaffected (EXP: $4.2 \pm 1.3$ vs. CON: $4.1 \pm 0.7 \mu \mathrm{M} ; p>0.05$; EXP: $1.00 \pm 0.48$ vs. CON: $0.89 \pm 0.28$ TS ratio; $p=0.77$, respectively); however, superoxidase dismutase activity was greater in the expedition group $\left(3.1 \pm 0.4 \mathrm{vs}\right.$. $\left.0.8 \pm 0.5 \mathrm{U} \mathrm{ml}^{-1} ; p<0.001\right)$.

Conclusion: These results indicate a modest improvement in metabolic and oxidative profiles with increased superoxidase dismutase levels, suggesting an antioxidative 
response to counteract the exercise-associated production of free radicals and reactive oxygen species during prolonged exercise, mimicking the effects from long-term training. Although improved antioxidant activity may lead to increased TL, the present exercise stimulus was insufficient to promote a positive cellular aging profile with concordant chromosomal changes in our healthy and young participants.

Keywords: prolonged exercise, oxidative stress, metabolism, telomeres, hormones

\section{INTRODUCTION}

Oxidative stress is the result of an imbalance between the production of free radicals (reactive oxygen species and nitrogen oxygen species), and a defense system of enzymatic and non-enzymatic antioxidants. This imbalance may cause lipid peroxidation (PEROX), as well as protein and DNA oxidation (Urso and Clarkson, 2003; Vollaard et al., 2005; Finaud et al., 2006), leading to metabolic and vascular dysfunctions, cancer, neurological diseases, and even cellular senescence (Xie and Huang, 2003; Cherkas et al., 2008; Puterman et al., 2010; Shammas, 2011; Denham et al., 2013). Mitochondria, at the complexes I, III, and IV, are believed to be a critical source of radicals (estimation of $0.15-5 \%$ of oxygen used is reduced to superoxide, hydrogen peroxide, and hydroxyl radicals, among others), along with nicotinamide adenine dinucleotide phosphate (NADPH) oxidase, phospholipase $\mathrm{A}_{2}\left(\mathrm{PLA}_{2}\right)$, and xanthine oxidase (Boveris and Chance, 1973; Ashton et al., 1998; St-Pierre et al., 2002; Powers et al., 2011).

Although the production of free radicals is known to originate from multiple organs such as the heart, lungs, and even blood (Powers and Jackson, 2008; Nikolaidis and Jamurtas, 2009), an increase in skeletal muscle activity, and its corresponding response to radical production, has led to many studies and reviews examining the effects of exercise on oxidative stress and health and performance (Vollaard et al., 2005; Finaud et al., 2006; Powers et al., 2011, 2016). While acute exercise, and the concurrent rise in skeletal muscle and mitochondrial activity, increases free radical generation, counterintuitively, regular training lowers systemic oxidative stress levels and improves antioxidative activity (Radak et al., 2008). Brites et al. (1999) observed an increase in plasma ascorbic and uric acid concentrations, $\alpha$-tocopherol, and superoxide dismutase (SOD), representing non-enzymatic and enzymatic antioxidant responses; in trained soccer players compared to sedentary controls. A 12-week training study by Miyazaki et al. (2001) further showed that not only antioxidative responses were influenced by training via higher levels of SOD and glutathione peroxidase (GXP), but oxidative stress was also reduced as neutrophilic superoxide anion and thiobarbituric acid reactive substances (TBARS) were lower post training. This was substantiated by Vincent et al. (2006) in older adults following a 6-month training program where lower levels of TBARS and PEROX as biomarkers of lipid peroxidation were shown. This upregulation of antioxidants is likely a key mechanism of the long-term health benefits of regular exercise. Regular physical activity or training is defined with significant resting sessions where the fluctuating redox status is able to initiate intracellular signaling of antioxidant protein expression. While we have a good understanding of oxidative stress under acute and chronic exercise models, its critical role in many neurological, immune, metabolic, and cardiovascular functions required thoughtful considerations for other types of exercise where redox status may be different.

Interestingly, lower oxidative stress, associated with exercise training, has also been linked to longer telomere length (TL) (Cherkas et al., 2008; Puterman et al., 2010; Shammas, 2011; Denham et al., 2013). Telomeres are repetitive, tandem, hexanucleotide DNA sequences that cap the end of chromosomes, protecting the genomic DNA from enzymatic degradation. They are important in cell division and replication, but are also used as a biological marker of cellular aging and senescence (Shin et al., 2008). TL in somatic cells shortens with age and telomeres that become too short are associated with subsequent cell death. Oxidative stress, induced by the inhibition of the antioxidant glutathione in cultured endothelial cells, has demonstrated acceleration in telomere erosion (Kurz et al., 2004). This suggests that regular exercise and its associated enhancement in anti-oxidants is protecting the degradation of the telomeres and mitigating the aging process and/or enhancing the health of individual cells. Denham et al. (2013) showed that endurance athletes had longer TL (T/S ratio) compared to an ageand sex-matched non-trained group. Six-month endurance or interval training interventions also seem to increase telomerase activity along with TL in lymphocytes, granulocytes, and other leukocytes in previously inactive individuals (Werner et al., 2018). Interestingly, Shin et al. (2008) did not observe an increase in TL following 6 months of training, despite greater GXP activity in erythrocytes.

While we have a good understanding of oxidative stress under acute and regular exercise models, given the critical role in neurological, immune, metabolic, and cardiovascular functions, the thorough understanding of the mechanisms of action for other types of exercise, where redox status may be different, require further study; particularly exercise patterns that are more relevant and generalizable to public application and interpretation. This analysis should include the examination of the associated metabolic and endocrine changes to contextualize the potential mechanisms of action.

One type of exercise that has been understudied to date is prolonged exercise across multiple days. Prolonged exercise is the continuous participation in low-to-moderate intensity activity for 6 or more hours daily, for multiple days. Limited research on the effects of prolonged exercise on oxidative stress have been performed despite its importance in occupational settings; for example, military excursions (Nindl et al., 2007), and wildland firefighting typically incur up to 14 days of continuous, 
low-to-moderate activity, 8-12 h/day or longer during an emergency (Robertson et al., 2016). Cases et al. (2006) examined antioxidant activity and expression in lymphocytes following a 5-day cycling event at moderate exercise intensity. An increase in SOD isoenzymes CuZn-SOD and Mn-SOD by 2.65- and 2.42fold, respectively, led to the conclusion that cellular adaptation in antioxidant activity was sufficient to counteract potential cellular damage from the 5-day prolonged oxidative stress. In contrast, a 5-day protocol of exhaustive swimming in Wistar rats demonstrated an increased oxidative stress via elevated levels of TBARS by $131 \%$ and conjugated dienes (CD) by $74 \%$, combined with decreased levels of SOD, catalase, glutathione peroxide, and glutathione-S-transferase (Thirumalai et al., 2011). During a 330-km, multi-day, ultra-marathon race, Mrakic-Sposta et al. (2015) reported an increase in reactive oxygen species and 8-hydroxy-2-deoxyguanosine (DNA damage). Borghini et al. (2015) demonstrated that, although TL was more preserved in endurance-trained athletes, it still was significantly shorter following the same $330-\mathrm{km}$ ultra-marathon race (post $0.86 \pm 0.4$ vs. pre $1.11 \pm 0.34 \mathrm{~T} / \mathrm{S}$ ratio).

In summary, despite its fundamental influence on cellular senescence and multiple pathologies, oxidative stress and antioxidant responses from exercise have been primarily examined following acute bouts and regular training only, with limited considerations for other types of exercise. Multi-day prolonged exercise may have similar total training workloads compared to months of classic endurance training protocols with lower resting times between bouts. Ultra-endurance prolonged events seem to indicate detrimental effects on TL along with greater oxidative stress, whereas chronic training demonstrates ambiguous results on TL with greater antioxidant activity. The impacts of multiday prolonged exercise on systemic oxidative stress responses are unclear, as are the associated metabolic, endocrine, and aging effects. Since multi-day prolonged endurance exercise can only be performed within the low- to moderate intensity exercise spectrum interspersed with moderate-vigorous bouts, and given that the majority of the population are not athletes, a focus on non-athlete, healthy adults would provide a greater scope of application to the general population. Therefore, the purpose of the present study was to determine if multi-day prolonged endurance exercise in healthy adults: (i) improves oxidative stress profiling; (ii) positively impacts metabolism; and (iii) influences TL.

\section{MATERIALS AND METHODS}

\section{Participants}

Twenty-five participants took part in the study in the month of June. Fifteen went on a 14-day, 260-km wilderness canoeing expedition, and 10 acted as controls (did not participate in expedition). Each participant was screened with a PAR-Q and a Health Screening form for cardiovascular, respiratory, or other conditions that could be aggravated by prolonged physical activity. None of the participants were on prescribed medications. Mean $( \pm S D)$ characteristics of the participants are presented in Table 1. Peak oxygen consumption ( $\left.\dot{\mathrm{V}} \cdot \mathrm{O}_{2 \text { peak }}\right)$, maximal
TABLE 1 | Participant's fitness characteristics (Mean $\pm S D$ ).

\begin{tabular}{lccc}
\hline & Control (6M:4F) & Expedition (11M:4F) & p-value \\
\hline Age (years) & $31.1 \pm 10.8$ & $23.5 \pm 7.4$ & $0.015^{*}$ \\
Height $(\mathrm{cm})$ & $174 \pm 9.7$ & $177.8 \pm 8.5$ & 0.375 \\
$A_{\mathrm{D}}\left(\mathrm{m}^{2}\right)$ & $1.86 \pm 0.21$ & $1.87 \pm 0.12$ & \\
$\dot{V} . \mathrm{O}_{2 \max }{ }^{1}\left(\mathrm{mlO}_{2} \cdot \mathrm{kg} \cdot \mathrm{min}^{-1}\right)$ & $47.1 \pm 9.0$ & $47.5 \pm 9.3$ & 0.745 \\
$\mathrm{HR}_{\max }($ beats.min & \\
$V_{\text {Emax }}\left(\mathrm{L} \cdot \mathrm{min}^{-1}\right)$ & $186 \pm 9$ & $196 \pm 10$ & $0.008^{*}$ \\
\hline
\end{tabular}

${ }^{1}$ Highest value from the maximal adapted incremental treadmill test. $A_{D}$, body surface area $=0.202 * W^{0.425} * H^{0.725}$ (DuBois, 1916); $\dot{V}_{2}$ max, maximal oxygen consumption; HRmax, maximal heart rate; $V_{\text {Emax }}$, maximal minute ventilation. *Age is significantly lower while HRmax is significantly greater in the expedition vs. the control group $(p<0.05)$.

ventilation $\left(V_{\mathrm{E}}\right)$, and maximal heart rate $\left(\mathrm{HR}_{\max }\right)$ were identified from the highest value achieved during the maximal incremental treadmill test described below. The study was performed according to the Declaration of Helsinki and was approved by the University Research Ethics Board (LUREB \#6011074) and all participants provided written informed consent.

\section{Experimental Protocol}

All participants (expedition group: EXP, $n=15$; controls: CON, $n=10$ ) arrived to the laboratory site $24 \mathrm{~h}$ before and $3 \mathrm{~h}$ after the expedition, in a fasted state, between 07:00 and 10:00 am, preceded by a 24 -h period without alcohol, caffeine, and tobacco. Fasting blood and saliva samples, as well as anthropometric data, were collected during these visits. Additional saliva samples were collected every 3 days.

The 14-day canoe trip was conducted on the Bloodvein River in northern Manitoba, Canada. The trip was initiated in Red Lake, Ontario, and concluded at Bloodvein Village, Manitoba. Participants paddled, portaged, hiked, swam, and performed other expedition-related tasks for travel and survival in the wilderness over a distance of approximately $260 \mathrm{~km}$ and over 14 days. Based on previous work (Shepard, 1987), and the physical activity intensity compendium (Ainsworth et al., 2000), daily energy expenditure during this period was expected to nearly double with exercise intensity ranging from low (camp chores), to moderate (flat water paddling), to high intensity (white water paddling, portaging, rapid swimming).

\section{Fitness, Health, and Anthropometric Measures}

Percentage body fat $(\% \mathrm{BF})$ was estimated from the sum of four skinfolds (Durnin and Womersley, 1974; Bloomer and FisherWellman, 2008), whereas body surface area was calculated from height $(\mathrm{H})$ and weight (W) as follows (DuBois, 1916):

$$
A_{\mathrm{D}}=0.202 \cdot \mathrm{W}^{0.425} \cdot \mathrm{H}^{0.725}
$$

Body mass index (BMI) was calculated as the mass divided by the square of body height. Waist circumference, hip circumference, as well as waist-to-hip ratio (WHR) were also measured and calculated. A hand-grip dynamometer was also used to provide an indirect measure of whole-body strength. Briefly, the subject 
held the dynamometer in the right hand, with the arm at right angles and the elbow by the side of the body and with the base resting on first metacarpal (heel of palm), while the handle was resting on middle of the four fingers. When ready the subject squeezed the dynamometer with maximum isometric effort, which was maintained for about $3 \mathrm{~s}$ with no other body movement was allowed. The participants performed three contractions with maximum efforts, each separated by $1 \mathrm{~min}$. The highest value was then used analysis. Resting mean arterial blood pressure (MAP) was calculated from assessed systolic and diastolic pressures using an automated blood pressure cuff on the arm (HEM-711AC, OMRON Healthcare Inc., Bannockburn, IL, United States). Maximal oxygen consumption was determined via a treadmill test which was initiated at a speed of $5 \mathrm{mph}$ at a grade of $0 \%$ for the first $3 \mathrm{~min}$. Subsequently, elevation was increased by $2.5 \%$ every $2 \mathrm{~min}$ until the participants reached exhaustion. Oxygen consumption $\left(\dot{\mathrm{V}} . \mathrm{O}_{2}\right)$, carbon dioxide release $\left(\dot{\mathrm{V}} \cdot \mathrm{CO}_{2}\right)$, and minute ventilation $\left(\dot{V}_{\mathrm{E}}\right)$ were assessed using an open-circuit ergospirometer (Vmax, Sensormedics, CA, United States) with a gas-mixing chamber. Before each trial, the gas analyzers were calibrated with air tanks containing $15 \% \mathrm{O}_{2}$ and $5 \% \mathrm{CO}_{2}$, respectively, for calibration of volumes, while the flow sensor was calibrated with a $3-\mathrm{L}$ syringe. A properly adjusted one-way Hans-Rudolph valve connected to a breathing tube was used in all tests to collect expired gases. A heart rate monitor (Polar H10, Kempele, Finland) was also used to assess resting heart rate and maximal heart rate during $\dot{\mathrm{V}} . \mathrm{O}_{2 \text { peak }}$ testing.

\section{Blood Sampling}

Push-button blood collection sets (21G) (BD Vacutainer, Franklin Lakes, NJ, United States) positioned in the antecubital vein were used to collect blood samples in 3.5-ml vacuum-sealed serum tubes with silicon coating (BD Vacutainer SST tubes, Franklin Lakes, NJ, United States), in 3-ml $\mathrm{K}_{2}$ EDTA whole blood tubes (BD Vacutainer Plus Plastic $\mathrm{K}_{2}$ EDTA tubes, Franklin Lakes, NJ, United States), and 4-ml heparin gel tube specially designed to isolate monocytes (peripheral blood mononuclear cells) (CPT Cell Preparation Tube, BD Vacutainer, Franklin Lakes, NJ, United States). Samples in serum tubes were given $30 \mathrm{~min}$ to coagulate as recommended by the manufacturer whereas plasma and CPT tube were centrifuged immediately. Blood samples were centrifuged at $3500 \mathrm{rpm}$ for $10 \mathrm{~min}(4100 \times \mathrm{g})$ followed by isolation of plasma and serum samples in Eppendorf tubes subsequently frozen at $-80^{\circ} \mathrm{C}$ for analyses. Monocyte samples from CPT tubes were immediately centrifuged at 1800 RFC for $15 \mathrm{~min}$. The white cell layer was pipetted into $15 \mathrm{ml}$ conical tube where it was mixed with $15 \mathrm{ml}$ of PBS for cell washing, and centrifuged again at $300 \mathrm{RFC}$ for $15 \mathrm{~min}$. The supernatant was removed and cells were washed again following the same procedure with $10 \mathrm{ml}$ of PBS. Cell pellets were moved to $2 \mathrm{ml}$ Eppendorf tubes, washed again, before supernatant was removed and cells were frozen at $-80^{\circ} \mathrm{C}$ for telomere analyses.

\section{Saliva Sampling}

Salivary samples were collected before, every 3 days during the expedition, and post expedition. Participants put a cotton swab in their mouth for a duration of approximately $1 \mathrm{~min}$ and then gently place the swab in a pre-labeled tube (Salivette ${ }^{\circledR}$, Saliva Examination, Germany). Pre and post samples were immediately frozen at $-80^{\circ} \mathrm{C}$ whereas samples collected during the expedition remained sealed and cooled at $\sim 5-10^{\circ} \mathrm{C}$ until the last laboratory visit where they were frozen at $-80^{\circ} \mathrm{C}$ (Chen et al., 1992). Samples from days 3, 6, 9, and 12 were collected prior to overnight sleeping following a water mouth rinse. Samples were later analyzed to assess saliva concentrations of cortisol.

\section{Oxidative Measures}

Oxidative stress was assessed via changes in malondialdehyde (MDA) (Nielsen et al., 1997). Plasma MDA was quantified by using the TBARS kit (Cayman Chemical Company, Ann Harbor, MI, United States). The kit uses the thiobarbituric acid (TBA) to react and adduct the MDA to form the MDA-TBA under high temperature $\left(90-100^{\circ} \mathrm{C}\right)$ and acidic conditions. Briefly, $100 \mu \mathrm{l}$ of plasma was mixed with $100 \mu \mathrm{l}$ of SDS solution and $4 \mathrm{ml}$ color reagent containing TBA, acetic acid, and sodium hydroxide. The samples were boiled in a water bath for $1 \mathrm{~h}$, cooled, and the absorbance was measured at $530 \mathrm{~nm}$ using fluorometric plate reader. The concentration of MDA was calculated and expressed as $\mu \mathrm{M}$. In addition, SOD was analyzed as an antioxidant marker protein (Castro and Freeman, 2001). The plasma total SOD activity $(\mathrm{U} / \mathrm{ml})$ was determined by SOD assay kit (Cayman Chemical Company, Ann Arbor, MI, United States) following the manufacturer's protocol.

\section{Relative Telomere Length}

DNA from leukocyte cell pellets were isolated from whole blood (BD Vacutainer ${ }^{(} \mathrm{CPT}^{\mathrm{TM}}$ ) using Nucleospin DNA isolation kit (MACHEREY-NAGEL GmbH \& Co. KG, Germany) (Stenbäck et al., 2019). Relative telomere length (RTL) was determined with qPCR using Cawthon's monochrome multiplex method (Cawthon, 2002, 2009). Briefly, $2 \mu$ l of DNA samples were amplified for 40 cycles, using either telomere or $\beta$-globin primers, and the FastStart Universal SYBR Green Master reagent (Roche) in $20 \mu \mathrm{l}$ final reaction volume. Reactions were run using telomere primers and beta-globin (single copy gene) primers on ABI 7300 real-time PCR system (Applied Biosystems, CA, United States) according to the following conditions: for telomere, $95^{\circ} \mathrm{C}$ for $10 \mathrm{~min}, 2$ cycles of $95^{\circ} \mathrm{C}$ for $15 \mathrm{~s}, 49^{\circ} \mathrm{C}$ for $15 \mathrm{~s}$ and 40 cycles of $95^{\circ} \mathrm{C}$ for $15 \mathrm{~s}, 60^{\circ} \mathrm{C}$ for $15 \mathrm{~s}, 70^{\circ} \mathrm{C}$ for $1 \mathrm{~min}$, and for $\beta$-globin, $95^{\circ} \mathrm{C}$ for $10 \mathrm{~min}, 40$ cycles of $95^{\circ} \mathrm{C}$ for $15 \mathrm{~s}$, $60^{\circ} \mathrm{C}$ for $1 \mathrm{~min}$ followed by a dissociation (or melt) curve for PCR product verification. The RTL was calculated using the $2^{-(\Delta \mathrm{Ct} 1-\Delta \mathrm{Ct} 2)}=2^{-\Delta \Delta \mathrm{Ct}}$, and expressed as $\mathrm{T} / \mathrm{S}$ ratio (telomere vs. single copy gene).

The primer sequences used for the qPCR amplifications were for telomere, Telg: ACA CTA AGG TTT GGG TTT GGG TTT GGG TTT GGG TTA GTG T, Telc: TGT TAG GTA TCC CTA TCC CTA TCC CTA TCC CTA TCC CTA ACA. The primers for $\beta$-globin were forward: CGG CGG CGG GCG GCG CGG GCT GGG CGG CTT CAT CCA CGT TCA CCTT G, reverse: GCC CGG CCC GCC GCG CCC GTC CCG CCG GAG GAG AAG TCT GCC GTT. 


\section{Metabolic Measures}

Serum total cholesterol (TCH), high-density lipoprotein (HDL), low-density lipoprotein (LDL), and triglycerides (TGs) provided a metabolic lipid profile of the participants pre and post expedition. Serum lipid profiles including TCH, HDL cholesterol (HDL-C), LDL cholesterol (LDL-C), and TG were measured by enzymatic method (Siemens Healthcare Diagnostics Inc., Erlangen, Germany). Serum glucose concentration was assayed by an enzymatic method involving hexokinase and glucose6-phosphate dehydrogenase (Siemens Healthcare Diagnostics Inc., Erlangen, Germany). Serum creatinine levels (Crn) were determined with the Jaffe reaction. Serum electrolytes sodium $(\mathrm{Na})$, potassium $(\mathrm{K})$, chloride $(\mathrm{CL})$, and urea measurements were performed using commercial kits (Siemens Healthcare Diagnostics Inc., Erlangen, Germany).

\section{Endocrine Measures}

Serum total testosterone (ECLIA, Roche Diagnostic, Mannheim, Germany) and plasma insulin levels (DIAsource immunoassays, S.A., Nivelles, Belgium) were analyzed using commercial immunoassay assays. The cortisol level was assessed from saliva samples with luminescence immunoassay kit (IBL International $\mathrm{GmbH}$, Hamburg, Germany) according to the manufacturer's instructions.

\section{Statistics}

Participant's age, height, $\dot{\mathrm{V}} \cdot \mathrm{O}_{2 \max }, \mathrm{HR}_{\max }$, and $\dot{\mathrm{V}}_{\mathrm{Emax}}$ were compared between EXP and CON using Student's $t$-test with a level of significance set at $p<0.05$. When normality or equal variance test failed, a Mann-Whitney Rank Sum test was performed. Weight, BMI, \%BF, waist circumference, hip circumference, WHR, grip strength, resting $\mathrm{HR}$, and resting MAP were analyzed via a two-way repeated measures ANOVA with time (levels: pre and post) and groups (levels: CON and EXP) as factors. Normality was tested via a Shapiro-Wilk test while the equal variance test via a Brown-Forsythe test. A level of significance was set at $p<0.05$. Saliva cortisol concentrations were analyzed via a two-way repeated measures ANCOVA, with age, gender, and fitness as covariates, to examine changes before, during, and after the expedition in both groups with group (levels: CON and EXP) and time (levels: baseline, 3 days, 6 days, 9 days, 12 days, and post) as factors. Blood serum fasting oxidative (MDA, SOD), endocrine (TES, INS, COR), and metabolic variables (creatinine, urea, glucose, $\mathrm{Na}^{+}, \mathrm{K}^{+}, \mathrm{Cl}^{-}$) and RTL from isolated blood mononuclear cells were analyzed via one-way ANCOVA analyses, with age, gender, fitness, and baseline values as covariates. Results were analyzed using STATA version 15 (Statacorp LLC, College Station, TX, United States).

\section{RESULTS}

\section{Fitness, Health, and Anthropometric Variables}

Post expedition, participants in the EXP group demonstrated lower weight $(-1.4 \mathrm{~kg})$, BMI $\left(-0.7 \mathrm{~m}^{2}\right), \% \mathrm{BF}(-1.2 \%)$, waist
TABLE 2 | Participant's anthropometric and health characteristics pre and post expedition in both the control and expedition group (Mean $\pm S D$ ).

\begin{tabular}{|c|c|c|c|c|}
\hline & \multicolumn{2}{|c|}{ Control } & \multicolumn{2}{|c|}{ Expedition } \\
\hline & Pre & Post & Pre & Post \\
\hline Weight (kg) & $72 \pm 14.5$ & $72.0 \pm 6.2$ & $72 \pm 6.2$ & $70.6 \pm 6.0^{*}$ \\
\hline $\mathrm{BMI}\left(\mathrm{kg} \cdot \mathrm{m}^{-2}\right)$ & $24 \pm 3.1$ & $23.6 \pm 3.1$ & $23 \pm 2.4$ & $22.3 \pm 2.2^{*}$ \\
\hline Body fat ${ }^{1}(\%)$ & $23 \pm 10.0$ & $22.5 \pm 9.5$ & $18 \pm 8.4$ & $16.8 \pm 9.0^{*}$ \\
\hline Waist circ. (cm) & $80 \pm 11.7$ & $80 \pm 12$ & $79 \pm 3.9$ & $76 \pm 4^{*}$ \\
\hline Hip circ. (cm) & $99 \pm 6.2$ & $100 \pm 6$ & $98 \pm 3.2^{\dagger}$ & $91 \pm 4^{*}$ \\
\hline Waist-hip ratio & $0.81 \pm 0.08$ & $0.80 \pm 0.09$ & $0.81 \pm 0.04$ & $0.83 \pm 0.05$ \\
\hline Grip strength (kg) & $83 \pm 21$ & $71 \pm 18^{*}$ & $92 \pm 22^{\dagger}$ & $100 \pm 19^{*}$ \\
\hline HR resting (b. $\min ^{-1}$ ) & $71 \pm 7$ & $64 \pm 8^{*}$ & $74 \pm 9$ & $63 \pm 9^{*}$ \\
\hline MAP resting $(\mathrm{mmHg})$ & $90 \pm 5$ & $92 \pm 7$ & $81 \pm 13^{\dagger}$ & $85 \pm 5$ \\
\hline
\end{tabular}

${ }^{1}$ Estimated from skinfolds (Durnin and Womersley, 1974). BMI, body mass index; circ, circumference; HR, heart rate; MAP, mean arterial pressure. * Significantly different between pre and post $(p<0.05)$. ${ }^{\dagger}$ Significantly different between control and expedition ( $p<0.05$ ).

circumference $(-3 \mathrm{~cm})$, hip circumference $(-7 \mathrm{~cm})$, and resting heart rate $\left(-11\right.$ beat $\left.\mathrm{min}^{-1}\right)$ while exhibiting greater grip strength $(+8 \mathrm{~kg})(p<0.01)$. The CON group had lower HR $\left(-7\right.$ beat $\left.\mathrm{min}^{-1}\right)$ and grip strength $(-12 \mathrm{~kg})(p<0.01)$ (Table 2$)$.

\section{Oxidative Stress Variables}

The enzymatic antioxidant activity from SOD was greater in the EXP group vs. CON $[2.9 \pm 0.31$ vs. $1.1 \pm 0.41, F(1,20)=7.127$, $p<0.001]$ and was not affected by age $(p=0.823)$, sex $(p>0.05)$, fitness $(p>0.05)$, or baseline values $(p>0.05)$ (Figure 1). Lipid peroxidation from MDA concentrations, however, was not significantly changed between groups $(p>0.05)$ (Figure 1). RTL was also not significantly different between groups $(p>0.05)$ (Figure 1).

\section{Endocrine Variables}

There were no statistical differences between EXP vs. CON for TES $\left(4.7 \pm 3.3\right.$ vs. $\left.3.65 \pm 3.14 \mathrm{ng} \mathrm{ml}^{-1}, p>0.05\right)$ (Figure 2). Cortisol also did not yield differences across time $(p>0.05)$ or conditions $(p>0.05)$, nor had any interactions between factors $(p>0.05)$ (Figure 3). However, INS fasting blood concentration was lower in the EXP condition $\left(5.0 \pm 2.46 \mu \mathrm{U} \mathrm{ml}^{-1}\right)$ compared to CON $\left(7.8 \pm 2.71 \mu \mathrm{U} \mathrm{ml}^{-1}\right)$ $[F(1,19)=8.148, p<0.05]$ and was not affected by any of the covariates (Figure 2).

\section{Metabolic Variables}

Creatinine and glucose were both lower in the EXP group vs. CON [Crn: $0.86 \pm 0.022$ vs. $1.00 \pm 0.025 \mathrm{mg} \mathrm{dl}^{-1}$, $F(1,19)=16.01, p<0.001$; glucose: $86 \pm 1.5$ vs. $96 \pm 1.8 \mathrm{mg} \mathrm{dl}^{-1}$, $F(1,19)=14.2, p<0.001]$ and were both influenced by baseline values [creatinine: $F(1,19)=24.8, p<0.001$; glucose: $F(1,19)=17.5, p<0.001]$ (Table 3). Urea concentration was not different between groups $(p>0.05)$ nor influenced by covariates. Concentrations of $\mathrm{Na}^{+}, \mathrm{K}^{+}$, nor $\mathrm{Cl}^{-}$were not different between EXP and CON $(p>0.05)$ (Table 3). 


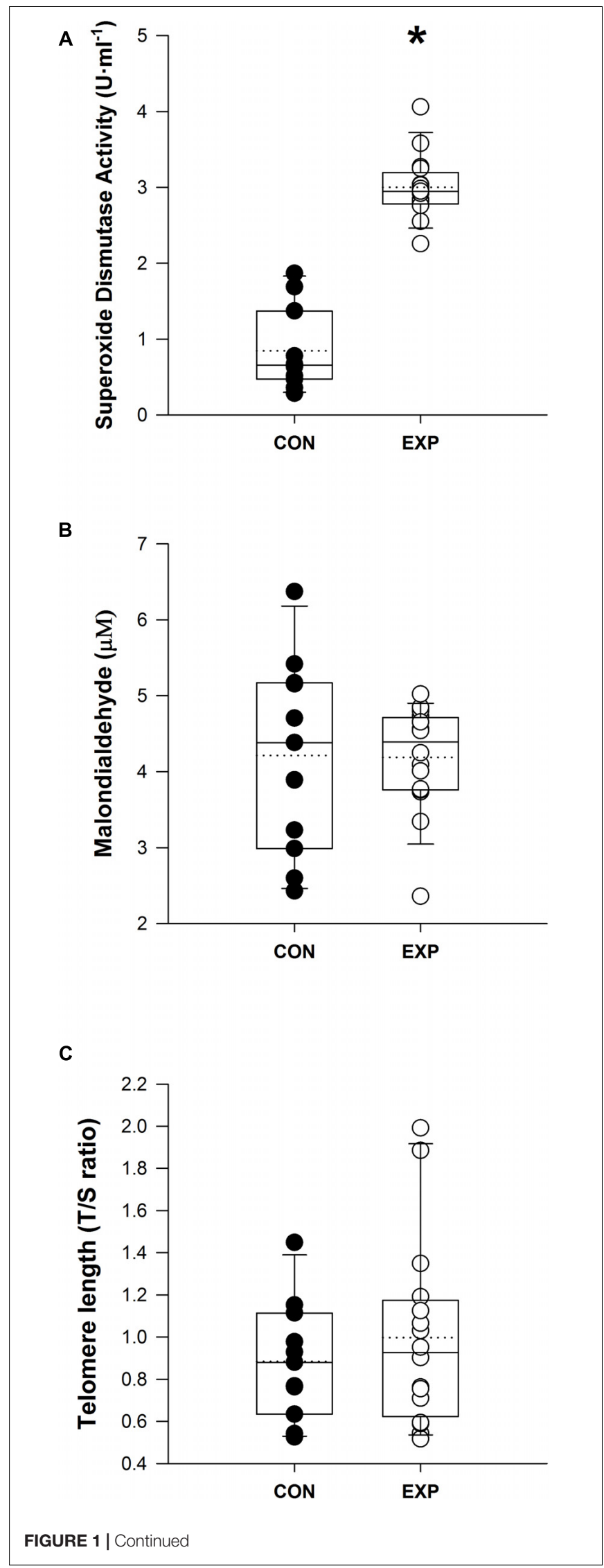

FIGURE 1 | (A) Blood plasma superoxide dismutase activity, (B) malondialdehyde, and (C) mononuclear cells relative telomere length values for each participant in the CON and EXP group. Boxplots line shows median value, dotted line shows the mean, and whiskers show the 5th and 95th percentile. *Superoxide dismutase is significantly greater in EXP vs. CON.

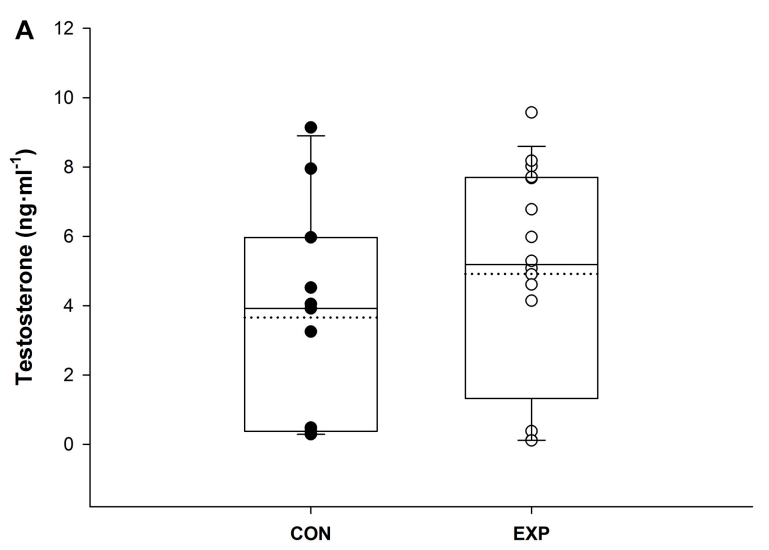

B

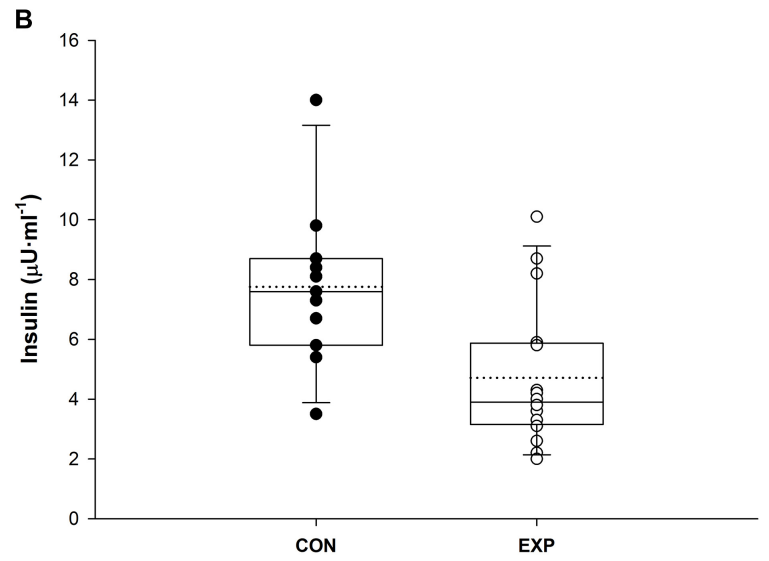

FIGURE 2 | (A) Blood plasma testosterone and (B) insulin concentration values for each participant in the CON and EXP group. Boxplots line shows median value, dotted line shows the mean, and whiskers show the 5th and 95th percentile.

\section{Lipid Profile}

Blood serum concentrations (Table 4) of CHOL, HDL, and LDL were not statistically different between groups except for TG which was lower in EXP $\left(72 \pm 5.1 \mathrm{mg} \mathrm{dl}^{-1}\right)$ compared to CON $\left(102 \pm 5.9 \mathrm{mg} \mathrm{dl}^{-1}\right)[F(1,19)=13.307, p<0.01]$ and was affected by baseline values $(F=38.4, p<0.001)$.

\section{DISCUSSION}

This present study examined the influence of multi-day prolonged low- to moderate-intensity endurance exercise on combined oxidative, metabolic, and endocrine profiles as well as telomere integrity in a healthy young human model. Our main findings were that multi-day prolonged low- to moderate-intensity endurance exercise: (1) modestly improved 


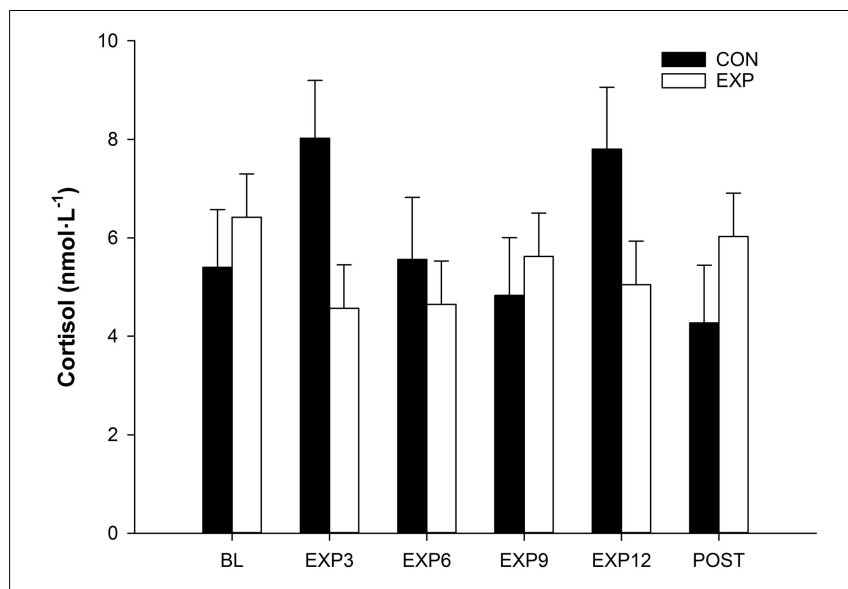

FIGURE 3 | Saliva cortisol concentration values for CON and EXP group at baseline (BL), three in expedition (EXP3), 6 days in expedition (EXP6), 9 days in expedition (EXP9), 12 days in expedition (EXP12), and post expedition (POST). Means \pm SEM.

lipid profile and energy metabolism with lower TG, glucose, creatinine, and insulin levels; (2) induced an increase in enzymatic antioxidative activity via greater SOD concentrations; (3) did not have an effect on oxidative stress per se as assessed via lipid peroxidation MDA levels; (4) did not result in notable changes in blood testosterone or salivary cortisol levels; and (5) did not result in notable changes in RTL. The expedition group, and not the control group, also had significant decreases in: weight, waist circumference, hip circumference, BMI, and increases in grip strength postexpedition. Altogether, these findings suggest a meaningful, but modest, improvement in both the metabolic and oxidative profiles without an influence on telomere integrity in those participating in multi-day prolonged endurance exercise within, primarily, the low- to moderate-intensity exercise spectrum. In the present study, metabolic improvements were substantiated by lower blood glucose, triglycerides, creatinine, and insulin concentrations as well as lower weight, BMI, and waist and hip circumference.
Although the definition of prolonged exercise is relatively wide, very few reports have examined the effects of exercise interventions beyond multiple days on oxidative stress. Prolonged or intense exercise induces a greater production of free radicals. Increased skeletal muscle activity with associated oxygen demands causes lower $\mathrm{O}_{2}$ levels (Richardson et al., 1995) and greater $\mathrm{CO}_{2}$ intracellular tension, this combined with an induced lower cellular $\mathrm{pH}$, creates a favorable milieu for oxidative stress at both the mitochondrial and cellular level (Arbogast and Reid, 2004). Vollaard et al. (2005) presented a clear association of acute exercise and increases in TBARS, and lipid oxidation in multiple studies with peak demand of $80 \mathrm{~km}$ of running (Kanter et al., 1988). Conversely, training studies, ranging from three to five times per week of exercise for 8-12 weeks, have reported a general improvement of the oxidative profile by both a reduction of free radical production (Leaf et al., 1999; Miyazaki et al., 2001; Radák et al., 2002; Bloomer and FisherWellman, 2008) and an increase in antioxidant activity (Brites et al., 1999; Radák et al., 2002). In the present study, multiday prolonged low- to moderate-intensity endurance exercise occurred over 14 consecutive days of $>7 \mathrm{~h}$ of predominantly low-moderate exercise daily. We found under these exercise conditions, oxidative stress was not present, as measured by MDA concentrations; however, there was a significant increase in antioxidant activity as measured via blood concentration of SOD. These findings partly support Cases et al. (2006) with elevated levels of CuZn-SOD and Mn-SOD 2.65- and 2.42 -fold. They, however, also noted an increase in carbonyls and reactive oxygen metabolites, both used as oxidative stress markers. Moreover, the endocrine responses in the present study revealed no changes in TES or COR but did detect decreases in serum INS post-expedition in the EXP group only. Prolonged exercise studies have generally demonstrated significant loss in adipose and skeletal muscle mass, as they are generally conducted under hypo-calorific diets, known to diminish TES and increase COR circulating levels (Nindl et al., 2007; Gagnon et al., 2011). We hypothesize that these differences are due to the differences in exercise regimes between groups as well as the study population, notably Nindl et al. (2007) and Gagnon et al. (2011) studies where hypocaloric diets were employed. Our results support this literature, as we also found improvements

TABLE 3 | Metabolic variables.

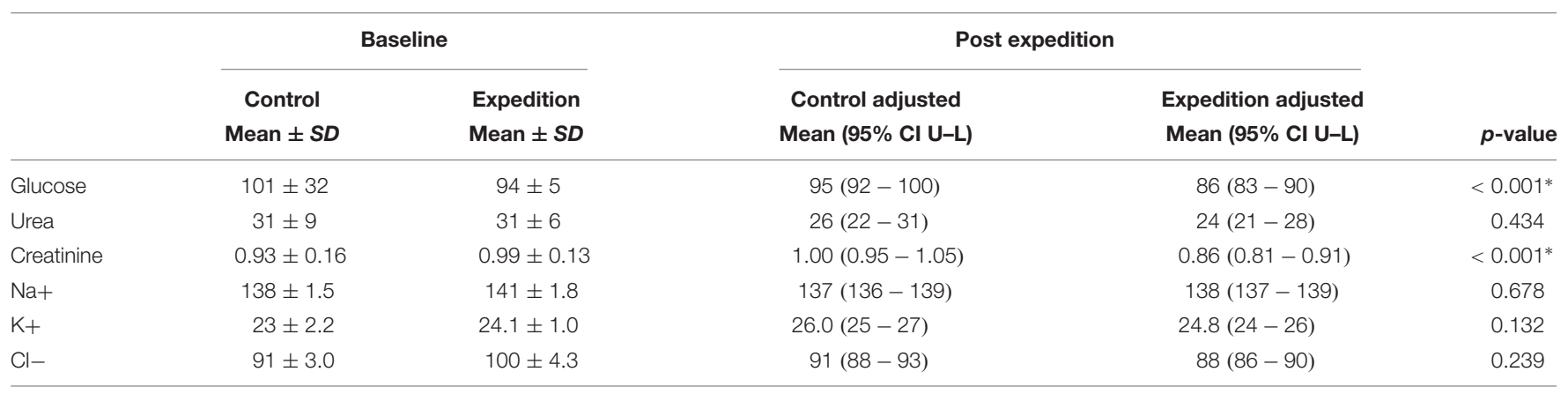

$\mathrm{Na}+$, sodium (mmol. $\left.\mathrm{L}^{-1}\right) ; \mathrm{K}+$, potassium (mmol. $\left.\mathrm{L}^{-1}\right)$; $\mathrm{Cl}$-, chloride $\left(m m o l . L^{-1}\right)$. Glucose, urea, and creatinine are expressed as $m g . d L^{-1}$. *Glucose and creatinine are significantly lower in the expedition vs. the control group post expedition $(p<0.05)$. 
in health characteristics of our participants, similar to previous reports where exercise loading exceeds a few days (Helge et al., 2006, 2008; Nindl et al., 2007; Gagnon et al., 2011).

\section{Oxidative Stress}

It has been well established that acute exercise induces increases in free radical production stemming from numerous pathways, including anions leaking from the mitochondrial electron transfer chain; due to increased $\mathrm{O}_{2}$ consumption and temperature, superoxide production from xanthine oxidase, $\mathrm{NADPH}$ oxidase from tissue damage, and auto-oxidation of catecholamines (Deaton and Marlin, 2003). While these processes may be influenced by training with lower free radical production, the increase in anti-oxidative activity from training seems to mitigate the rise in free radicals during exercise more effectively, lowering redox status, and allowing more strenuous physical work to be performed (Leaf et al., 1999). Miyazaki et al. (2001) observed a decrease in neutrophil superoxide anion and TBARS after exhaustive exercise following 12 weeks of running, 5 days per week for $60 \mathrm{~min}$ at $80 \%$ of $\mathrm{VO}_{2 \max }$; but no differences in antioxidant enzymes activity. Interestingly, SOD, GXP, and catalase were elevated post training, at rest. A similar finding was obtained by Brites et al. (1999) who found that a group of soccer players had higher levels of antioxidant capacity, ascorbic acid, uric acid, $\alpha$-tocopherol, and SOD compared to sedentary controls following an overnight fast. In our study, the EXP group arrived at the lab approximately $3 \mathrm{~h}$ after exiting the river, providing a resting sample for analyses, and exhibited greater anti-oxidative activity compared to our CON group. Similar to Miyazaki's training, our results showed no changes in oxidative stress at rest following the exercise intervention, providing some insights on an analogous role of multi-day prolonged exercise compared to long-term training. Cases et al. (2006) reported an increase in both oxidative and antioxidative responses after prolonged exercise and oxidative stress but sampling occurred just after by two exhaustive cycling events (164.5 and $166.3 \mathrm{~km}$ performed in 255 and $234 \mathrm{~min}$, respectively); highlighting the potential transient nature of these increases. Although the above-mentioned exercise designs contain prolonged exercise, a distinction should be established where our exercise intervention did not lead to physical exhaustion on a daily basis, but rather relied on lower exercise intensities (low and moderate) for a longer duration (69 h daily).

\section{Telomere Influence}

Telomeres contain genomic information necessary for cell preservation and replication. TL and its relationship with cellular senescence has been investigated, along with many influencing factors such as lifestyle, diet, psychological stress, and exercise (Kurz et al., 2004; Cherkas et al., 2008; Puterman et al., 2010; Shammas, 2011). Acute exercise has been shown to induce a measurable decrease in TL in 15 athletes following an ultra-marathon race (Borghini et al., 2015). However, $30 \mathrm{~min}$ of running was shown to be sufficient to upregulate 56 miRNAs as well as telomerasereverse transcriptase (TERT) and sirtuin-6 (SIRT6) mRNA expression from white blood cells; both of these are critical in telomere maintenance and repair (Chilton et al., 2014) suggesting that regular low-to-moderate intensity has beneficial effects on TL. Short bouts of exercise can induce molecular signaling response for increased TL, whereas acute oxidative stress negates, at least briefly, that response. No studies have examined the specific time scale and intensity required to obtain benefits in TL from exercise. There is a gap between our study and others pointing toward unchanged TL even after 6 months of training (Shin et al., 2008), and retrospective work associating TL to long-term physical activity (Kurz et al., 2004; Cherkas et al., 2008; Puterman et al., 2010; Shammas, 2011). Although Werner et al. (2018) indicated that 6 months of endurance (with a total of $<60 \mathrm{~h}$ of moderate-intensity exercise) or interval training may be sufficient to increase TL. Our study, instead, required approximately $100-120 \mathrm{~h}$ of low- to moderateintensity exercise, which should have been sufficient of a workload to induce a change in TL. While it is difficult to determine what caused the lack of change, intra-individual phenotype and genotype factors may result requiring years of physical activity to observe quantitative and detectable changes (Shin et al., 2008).

\section{Endocrine and Metabolic Responses}

Prolonged physical stress, such as during strenuous exercise/sleep and energy deficit, induces increases in COR, while decreases TES (Nindl et al., 2007; Gagnon et al., 2011). Our measure of oxidative stress, MDA, did not change between groups after the expedition, indicating sufficient rest and food intake during the expedition. These factors limit the influences of stress, and likely explain the lack of differences for those two hormones. Literature highlighting the benefits of being exposed to nature on human health may also contribute to this study's findings. Beil and Hanes (2013) who examined the effect of visitation to different types of outdoor environments observed lower cortisol levels. Interestingly, we did find changes in the insulin response which was lower in the EXP group. The relationship between oxidative stress, insulin signaling, and resistance has been described (Henriksen et al., 2011; Rains and Jain, 2011). Insulin resistance is induced by increased oxidative stress via the activation of transcription factors (e.g., NF-kB, HIF-1) and the gene expression of pro-inflammatory cytokines (e.g., IL-6, TNF- $\alpha$ ), as well as mitochondrial dysfunction (Bloch-Damti and Bashan, 2001; Lowell and Shulman, 2005). Both mechanistic pathways rely on the activation or serine/threonine kinases, which decreases glucose transporter-4 (GLUT-4) expression, and consequently decreases the uptake of glucose into the cell and increase circulating glucose. Helge et al. (2006) examined an increase in GLUT-4 following a 42-day Greenland icecap crossing in the deltoid muscle with no concomitant change in INS circulating levels, nor in insulin sensitivity markers (HOMA, $\mathrm{DI}, \mathrm{AIR}_{\mathrm{g}}$ ). Interestingly, this was coupled with a decrease in lean body mass and a related decrease in aerobic fitness. Our 
TABLE 4 | Lipid profile.

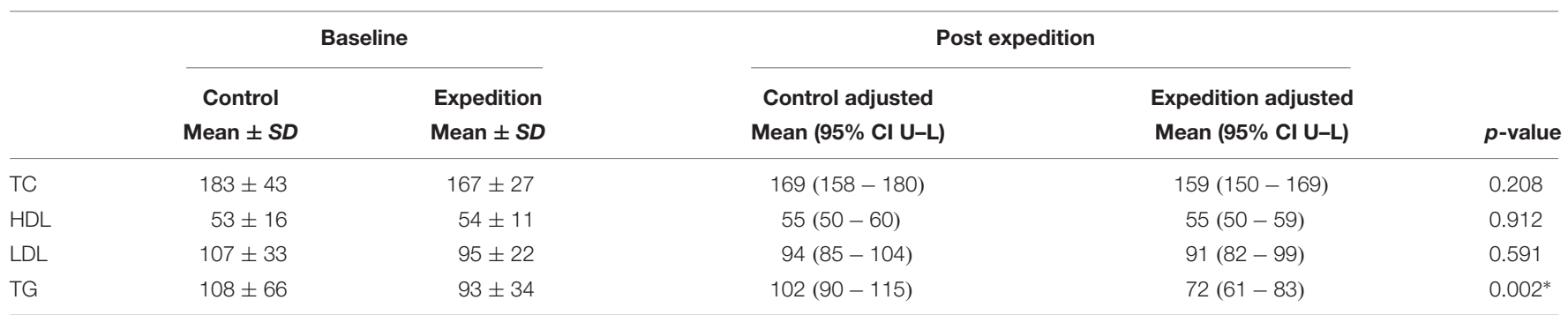

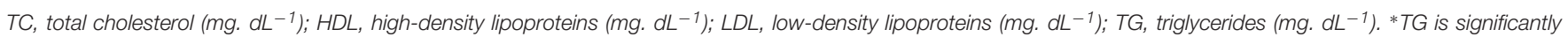
lower in the expedition vs. the control group post expedition $(p<0.05)$.

participants lost a mean $1.4 \mathrm{~kg}$ of mass and $1.2 \%$ of $\mathrm{BF}$, for a loss of $0.5 \mathrm{~kg}$ of lean mass. This is not comparable to the nearly $3 \mathrm{~kg}$ of Helge et al. (2006), suggesting that muscle loss is not an important factor in the upregulation of GLUT-4. Houmard et al. (2004) examined exercise volume, duration, and intensity on insulin sensitivity and found that, across the three components, duration had the largest effect on insulin sensitivity. The multi-day prolonged exercise from our intervention could have consequently increased insulin sensitivity, lowering the need for insulin secretion and maintaining a lower glucose level; all indicators of improved metabolic health.

Finally, we also measured a lower level of Crn in this study. It is unlikely that this effect is due to enhanced renal functions and clearance post-expedition; given the age and health of our study population. We hypothesize this effect is due to two changes in metabolism from the activity itself. First, we believe that there was likely an enhanced, continuous uptake of amino acids by the muscles cause a decline in all amino acids including Crn. This is supported by both the measured increase in grip strength as well as our estimated increase in total muscle mass. In addition, under these conditions of prolonged, low-to-moderate intensity exercise, the cells would enhance the protective mechanisms to prevent muscle losses, this would include a metabolic shift toward enhanced lipid metabolism and suppressed protein and carbohydrate metabolism. This hypothesis is supported by the measured decreased in fat tissue and overall weight loss (Horowitz and Klein, 2000). It is well known that endurance training induces a shift toward lipid metabolism. Interestingly, Tunstall et al. (2002) presented a clear activation of lipid metabolism gene expression after only 9 days of endurance training with increases in FAT/CD36, CPT1, PPAR $\alpha, \operatorname{PPAR} \gamma$, and others. Our participants were physically active for 14 days, and would have expressed a similar shift.

\section{Considerations}

Some limitations were identified in this study. The weight loss observed following the expedition in the EXP group $(-1.4 \mathrm{~kg})$ was minimal but nonetheless demonstrated that multi-day prolonged exercise was performed under hypocaloric conditions, despite our best attempt to maintain an equicaloric diet for our participants. Hypocaloric diets during multi-day prolonged exercise have previously been associated with detrimental health effects (i.e., dysregulated lipids concentrations, muscle mass loss), and a noticeable decrease in circulating total and free TES levels (Nindl et al., 2007; Gagnon et al., 2011). These findings were not present in the current study, suggesting that nutritional intake was sufficient to maintain exercise demands without compromising endocrine and health measures. This is further supported by the improvement in grip strength detected in the EXP group. Furthermore, our data collection platform included MDA as a lipid peroxidation marker for oxidation stress, yet the inclusion of protein (carbonyl and/or carbonyl/protein ratio) as well as DNA (8-hydroxy-deoxyguanosine) oxidative markers would have greatly contributed to understanding of the present results. Similarly, assessing enzymatic antioxidants such as catalase and glutathione as well as non-enzymatic antioxidants as variables would have benefited this study. Moreover, while our groups were composed of both males and females, only three females were part of each group. Even if sex was used as a co-variate within our analyses, critical physiological differences between males and females may limit the present interpretation of some results, particularly in the case of TES concentrations since the effects of exercise were reflected very differently between both the sexes. Finally, we were unable to perform a $\dot{V} \cdot \mathrm{O}_{2 \text { max }}$ test post expedition, which would have determined whether cardiorespiratory capacities were influenced by the exercise design, and whether a training effect occurred.

\section{Implications and Perspectives}

Many studies have examined the use of antioxidants (e.g., polyphenolics and vitamins $\mathrm{E}$ and $\mathrm{C}$ ) (Morillas-Ruiz et al., 2006) during or following exercise and training, and their effects on muscle damage, inflammation, or the maintenance of physical performance parameters such as skeletal muscle fiber contractile properties, excitation contraction coupling, power output, and fatigue (Alessio et al., 2000; Clarkson and Thompson, 2000; Kubukeli et al., 2002). While this may be useful in an acute exercise setting, the blunting of the increase in free radicals prevents the activation of gene expression signaling pathways of exercise-induced mitochondrial biogenesis and enzymatic antioxidants defense system (Nieman et al., 2010; Petersen et al., 2012). The redox status influences telomere state and not solely the generation of 
free radicals, and the use of antioxidants which could influence telomere integrity. Future investigations should consider the long-term influences of certain antioxidant supplements on longevity and health.

\section{CONCLUSION}

The present study offered insight on the oxidative stress response, antioxidant response, metabolic and endocrine changes, as well as telomere influence from exposure to multi-day prolonged low- to mid-intensity endurance exercise, in a healthy, but nonathlete population. Our main findings are that this type of exercise, without exhaustion, modestly improved lipid profile and energy metabolism while increasing enzymatic antioxidative activity, while have no detectable changes in resting endocrine responses or in RTL; mimicking similar redox balance benefits from training ranging from weeks to months.

\section{ETHICS STATEMENT}

This study was carried out in accordance with the recommendations of the Canadian Tri-Council research guidelines and the Research Ethics Board of Laurentian University, with written informed consent from all subjects. All subjects gave written informed consent in accordance with the Declaration of Helsinki. The protocol was approved by the Research Ethics Board of Laurentian University.

\section{REFERENCES}

Ainsworth, B. E., Haskell, W. L., Whitt, M. C., Irwin, M. L., Swartz, A. M., Strath, S. J., et al. (2000). Compendium of physical activities an update of activity codes and MET intensities. Med. Sci. Sports Exerc. 32, S498-S516.

Alessio, H. M., Hagerman, A. E., Fulkerson, B. K., Ambrose, J., Rice, R. E., and Wiley, R. L. (2000). Generation of reactive oxygen species after exhaustive aerobic and isometric exercise. Med. Sci. Sports Exer. 32, 1576-1581. doi: 10.1097/00005768-200009000-00008

Arbogast, S., and Reid, M. B. (2004). Oxidant activity in skeletal muscle fibers is influenced by temperature, $\mathrm{CO} 2$ level, and muscle-derived nitric oxide. Am. J. Physiol. Regul. Integr. Comp. Physiol. 201, 2379-2388. doi: 10.1152/ajpregu. 00072.2004

Ashton, T., Rowlands, C. C., Jones, E., Young, I. S., Jackson, S. K., and Davies, B. (1998). Electron spin resonance spectroscopic detection of oxygen-centred radicals in human serum following exhaustive exercise. Eur. J. Appl. Physiol. Occup. Physiol. 77, 498-502. doi: 10.1007/s004210050366

Beil, K., and Hanes, D. (2013). The influence of urban natural and built environments on physiological and psychological measures of stress-A pilot study. Int. J. Environ. Res. Public Health. 10, 1250-1267. doi: 10.3390/ ijerph10041250

Bloch-Damti, A., and Bashan, N. (2001). Proposed mechanism for the induction of insulin resistance by oxidative stress. Antioxid. Redox Signal. 7, 1553-1567. doi: 10.1089/ars.2005.7.1553

Bloomer, R. J., and Fisher-Wellman, K. H. (2008). Blood oxidative stress biomarkers: influence of sex, exercise training status, and dietary intake. Gend. Med. 5, 218-228. doi: 10.1016/j.genm.2008.07.002

Borghini, A., Giardini, G., Tonacci, A., Mastorci, F., Mercuri, A., MrakicSposta, S., et al. (2015). Chronic and acute effects of endurance training on telomere length. Mutagenesis 30, 711-716. doi: 10.1093/mutage/ gev038

\section{AUTHOR CONTRIBUTIONS}

DG, SD, SR, and $\mathrm{K}-\mathrm{HH}$ designed the study and conducted the experiment. SM, VS, and JW performed the biochemical analyses of blood and saliva samples. All authors offered critical feedback on results and findings and contributed to the writing of the manuscript.

\section{FUNDING}

This study was partly supported by a Travel Research Grant from the University of Oulu and a Laurentian University Research Grant (LURF\#6008209). Dr. DG was supported by a Natural Sciences and Engineering Research Council of Canada Discovery Grant (NSERC\#2016-060883).

\section{ACKNOWLEDGMENTS}

We would like to thank Remi Kamakura for her support in analyzing saliva cortisol samples, and Dr. Bruce Oddson for support with the statistical analyses. We would also like to acknowledge the help of the Outdoor Adventure Leadership Team at the Laurentian University in helping us coordinate and execute this study, in particular Mr. Jim Little, Mr. Sebastien Nault, Ms. Bryn Wilgress, Ms. Emily Morgan, and Ms. Gillian Rick and also thank all of the participants who took part in this very challenging study.

Boveris, A., and Chance, B. (1973). The mitochondrial generation of hydrogen peroxide: general properties and effect of hyperbaric oxygen. Biochem. J. 134, 707-716. doi: 10.1042/bj1340707

Brites, F. D., Evelson, P. A., Christiansen, M. G., Nicol, M. F., Basilico, M. J., Wikinski, R. W., et al. (1999). Soccer players under regular training show oxidative stress but and improved plasma antioxidant status. Clin. Sci. 96, 381-385. doi: 10.1042/cs0960381

Cases, N., Sureda, A., Mastre, I., Tauler, P., Aguilo, A., Cordova, A., et al. (2006). Response of antioxidant defenses to oxidative stress induced by prolonged exercise: antioxidant enzyme gene expression in lymphocytes. Eur. J. Appl. Physiol. 98, 263-269. doi: 10.1007/s00421-006-0273-y

Castro, L., and Freeman, B. A. (2001). Reactive oxygen species in human health and disease. Nutrition 17, 161-165.

Cawthon, R. M. (2002). Telomere measurement by quantitative PCR. Nucleic Acids Res. 30, e47.

Cawthon, R. M. (2009). Telomere length measurement by a novel monochrome multiplex quantitative PCR method. Nucleic Acids Res. 37;e21. doi: 10.1093/nar/ gkn1027

Chen, Y. M., Cintron, N. M., and Whison, P. A. (1992). Long-term storage of saliva cortisol samples at room temperature. Clin. Chem. 38:304.

Cherkas, L. F., Hunkin, J. L., Kato, B. S., Richards, J. B., Gardner, J. P., Surdulescu, G. L., et al. (2008). The association between physical activity in leisure time and leukocyte telomere length. Arch. Intern. Med. 168, 154-158. doi: 10.1001/ archinternmed.2007.39

Chilton, W. L., Marques, F. Z., West, J., Kannourakis, G., Berzins, S. P., O’Brien, B. J., and Charchar, F. J. (2014). Acute exercise leads to regulation of telomere-associated genes and microRNA expression in immune cells. PLoS One 9:e92088. doi: 10.1371/journal.pone.0092088

Clarkson, P. M., and Thompson, H. S. (2000). Antioxidants: what role do they play in physical activity and health? Am. J. Clin. Nutrit. 72, 637s-646s. doi: 10.1093/ajcn/72.2.637S 
Deaton, C. M., and Marlin, D. J. (2003). Exercise-associated oxidative stress. Clin. Tech. Equine Pract. 2, 278-291. doi: 10.1053/S1534-7516(03) 00070-2

Denham, J., Nelson, C. P., O’Brien, B. J., Nankervis, S. A., Denniff, M., Harvey, J. T., et al. (2013). Longer leukocyte telomeres are associated with ultra-endurance exercise independent of cardiovascular risk factors. PLoS One 8:e69377. doi: 10.1371/journal.pone.0069377

DuBois, D. F. (1916). A formula to estimate the approximate surface area if height and body mass be known. Arch. Intern. Med. 17, 863-871.

Durnin, J. V. G. A., and Womersley, J. (1974). Body fat assessed from total body density and its estimation from skinfold thickness: measurements on 481 men and women aged from 16 to 72 years. Br. J. Nutr. 32, 77-97. doi: 10.1079/ bjn 19740060

Finaud, J., Lac, G., and Filaire, E. (2006). Oxidative stress. Sports Med. 36, 327-358.

Gagnon, D. D., Pullinen, T., Karinen, H., Rintamäki, H., and Kyröläinen, H. (2011). Recovery of hormonal, blood lipid, and hematological profiles from a North Pole expedition. Aviar. Space Env. Med. 82, 1110-1117. doi: 10.3357/asem.3016.2011

Helge, J. W., Damsgaard, R., Overgaard, K., Andersen, J. L., Donsmark, M., Dyrskog, S. E., et al. (2008). Low-intensity training dissociates metabolic from aerobic fitness. Scand. .J. Med. Sci. Sports 18, 86-94. doi: 10.1111/j.1600-0838. 2006.00604.x

Helge, J. W., Overgaard, K., Damsgaard, R., Sorensen, K., Andersen, J. L., and Dyrskog, S. E. (2006). Repeated prolonged whole-body low-intensity exercise: effects on insulin sensitivity and limb muscle adaptation. Metab. Clin. Exp. 55, 217-223. doi: 10.1016/j.metabol.2005.08.015

Henriksen, E. J., Diamond-Stanic, M. K., and Marchionne, E. M. (2011). Oxidative stress and the etiology of insulin resistance and type 2 diabetes. Free Rad. Biol. Med. 51, 993-999. doi: 10.1016/j.freeradbiomed.2010. 12.005

Horowitz, J. F., and Klein, S. (2000). Effect of the volume and intensity of exercise training on insulin sensitivity. J. Appl. Physiol. 106, 101-106. doi: 10.1152/ japplphysiol.00707.2003

Houmard, J. A., Tanner, C. J., Slentz, C. A., Duscha, B. D., McCartney, J. S., and Kraus, W. E. (2004). Effect of the volume and intensity of exercise training on insulin sensitivity. J. Appl. Physiol. 96, 101-106. doi: 10.1152/japplphysiol. 00707.2003

Kanter, M. M., Lesmes, G. R., Kaminski, L. A., La Ham-Saeger, J., and Nequin, N. D. (1988). Serum creatine kinase and lactate dehydrogenase changes following an eighty kilometer race: relationship to lipid peroxidation. Eur. J. Appl. Physiol. Occup. Physiol. 57, 60-63. doi: 10.1007/bf00691239

Kubukeli, Z. N., Noakes, T. D., and Dennis, S. C. (2002). Training techniques to improve endurance exercise performances. Sports Med. 32, 489-509. doi: 10.2165/00007256-200232080-00002

Kurz, D. J., Decary, S., Hong, Y., Triver, E., Akhmedov, A., and Erusalimsky, J. D. (2004). Chronic oxidative stress compromises telomere integrity and accelerates the onset of senescence in human endothelial cells. J. Cell Sci. 117, 2417-2426. doi: $10.1242 /$ jcs.01097

Leaf, D. A., Kleinman, M. T., Hamilton, M., and Deitrick, R. W. (1999). The exercise-induced oxidative stress paradox: the effects of physical exercise training. Am. J. Med. Sci. 317, 295-300. doi: 10.1097/00000441-19990500000005

Lowell, B. B., and Shulman, G. I. (2005). Mitochondrial dysfunction and type 2 diabetes. Science 307, 384-387. doi: 10.1126/science.1104343

Miyazaki, H., Oh-ishi, S., Ookawara, T., Kizaki, T., Toshinai, K., Ha, S., et al. (2001). Strenuous endurance training in humans reduced oxidative stress following exhausting exercise. Eur. J. Appl. Physiol. 84, 1-6. doi: 10.1007/s004210000342

Morillas-Ruiz, J. M., Villegas Garcia, J. A., Lopez, F. J., Vidal-Guevara, M. L., and Zafrilla, P. (2006). Effects of polyphenolic antioxidants on exercise-induced oxidative stress. Clin. Nutri. 25, 444-453. doi: 10.1016/j.clnu.2005.11.007

Mrakic-Sposta, S., Gussoni, M., Moretti, S., Pratali, L., Giardini, G., Tacchini, P., et al. (2015). Effects of mountain ultra-marathon running on ros production and oxidative damage by micro-invasive analytic techniques. PLoS One 10:e0141780. doi: 10.1371/journal.pone.0141780

Nielsen, F., Mikkelsen, B. B., Nielsen, J. B., Andersen, H. R., and Grandjean, P. (1997). Plasma malondialdehyde as biomarker for oxidative stress: reference interval and effects of life-style factors. Clin. Biochem. 43, 1209-1214.
Nieman, D. C., Williams, A. S., Shanely, R. A., Jin, F., McAnulty, S. R., Triplett, N. T., et al. (2010). Quercetin's influence on exercise performance and muscle mitochondrial biogenesis. Med. Sci. Sports Exerc. 42, 338-345. doi: 10.1249/ MSS.0b013e3181b18fa3

Nikolaidis, M. G., and Jamurtas, A. Z. (2009). Blood as a reactive species generator and redox status regulator during exercise. Arch. Biochem. Biophys. 490, 77-84. doi: 10.1016/j.abb.2009.08.015

Nindl, B. C., Barnes, B. R., Alemany, J., Frykman, P. N., Shippee, R. L., and Friedl, K. E. (2007). Physiological consequences of U.S. Army ranger training. Med. Sci. Sports Exerc. 39, 1380-1387. doi: 10.1249/mss.0b013e318067e2f7

Petersen, A. C., McKenna, M. J., Medved, I., Murphy, K. T., Brown, M. J., Della Gatta, P., et al. (2012). Infusion with the antioxidant $\mathrm{N}$-acetylcysteine attenuates early adaptive responses to exercise in human skeletal muscle. Acta Physiol. 204, 382-392. doi: 10.1111/j.1748-1716.2011.02344.x

Powers, S. K., and Jackson, M. J. (2008). Exercise-induced oxidative stress: cellular mechanisms and impact on muscle force production. Physiol. Rev. 88, 12431276. doi: 10.1152/physrev.00031.2007

Powers, S. K., Nelson, W. B., and Hudson, M. (2011). Exercise-induced oxidative stress in humans: cause and consequences. Free Rad. Biol. Med. 51, 942-950. doi: 10.1016/j.freeradbiomed.2010.12.009

Powers, S. K., Radak, Z., and Ji, L. L. (2016). Exercise-induced oxidative stress: past, present and future. J. Physiol. 594. 18, 5081-5092. doi: 10.1113/JP270646

Puterman, E., Lin, J., Blackburn, E., O’Donovan, A., Adler, N., and Epel, E. (2010). The power of exercise: buffering the effect of chronic stress on telomerase length. PLoS One 5:e10837. doi: 10.1371/journal.pone0010837

Radak, Z., Chung, H. Y., and Goto, A. (2008). Systemic adaptation to oxidative challenge induced by regular exercise. Free Radic. Biol. Med. 44, 153-159. doi: 10.1016/j.freeradbiomed.2007.01.029

Radák, Z., Naito, H., Kaneko, T., Tahara, S., Nakamoto, H., Takahashi, R., et al. (2002). Exercise training decreases DNA damage and increases DNA repair and resistance against oxidative stress of proteins in aged rat skeletal muscle. Pflügers Arch. 445, 273-278. doi: 10.1007/s00424-002-0918-6

Rains, J. L., and Jain, S. K. (2011). Oxidative stress, insulin signaling, and diabetes. Free Rad. Biol. Med. 50, 567-575. doi: 10.1016/j.freeradbiomed.2010.12.006

Richardson, R. S., Noyszewski, E. A., Kendrick, K. F., Leigh, J. S., and Wagner, P. D. (1995). Myoglobin O2 desaturation during exercise: evidence of limited O2 transport. J. Clin. Invest. 96, 1916-1926. doi: 10.1172/jci118237

Robertson, A. H., Larivière, C., Leduc, C. R., McGillis, Z., Eger, T., Godwin, A., et al. (2016). Novel tools in determining the physiological demands and nutritional practices of Ontario Firefighters during fire deployment. PloS One 12:e0169390. doi: 10.1371/journal.pone.0169390

Shammas, M. A. (2011). Telomeres, lifestyle, cancer, and aging. Curr. Opin. Nutr. Metab. Care. 14, 28-34. doi: 10.1097/MCO.0b013e32834121b1

Shepard, R. J. (1987). Science and Medicine of canoeing and kayaking. Sports Med. 4, 19-33. doi: 10.2165/00007256-198704010-00003

Shin, Y. A., Lee, J. H., Song, W., and Jun, T. W. (2008). Exercise training improves the antioxidant enzyme activity with no change in telomere length. Mech. Aging 129, 254-260. doi: 10.1016/j.mad.2008.01.001

Stenbäck, V., Jagalur Mutt, S., Leppäluoto, J., Gagnon, D. D., Mäkelä, K., Jokelainen, J., et al. (2019). Association of physical activity with telomere length among elderly adults - the Oulu cohort. Front. Physiol. 10:444. doi: 10.3389/ fphys.2019.00444

St-Pierre, J., Buckingham, J. A., Roebuck, S. J., and Brand, M. D. (2002). Topology of superoxide production from different sites in the mitochondrial electron transport chain. J. Biol. Chem. 277, 44784-44790. doi: 10.1074/jbc.m207217200

Thirumalai, T., Theresa, S. V., Elumalain, E. K., and David, E. (2011). Intense and exhaustive exercise induce oxidative stress in skeletal muscle. Asian Pac. J. Trop. Dis. 1, 63-66. doi: 10.1016/s2222-1808(11)60016-9

Tunstall, R. J., Mehan, K. A., Wadley, G. D., Collier, G. R., Bonen, A., Hargreaves, M., et al. (2002). Exercise training increases lipid metabolism gene expression in human skeletal muscle. Am. J. Physiol. Endocrinol. Metab. 283, E66-E72. doi: 10.1152/ajpendo.00475.2001

Urso, M. L., and Clarkson, P. M. (2003). Oxidative stress, exercise, and antioxidant supplementation. Toxicology 189, 41-54. doi: 10.1016/s0300-483x(03)00151-3

Vincent, H. K., Bouguigon, C., and Vincent, K. R. (2006). Resistance training lowers exercise-induced oxidative stress and homocysteine levels in overweight and obese older adults. J. Physiol. 14, 1921-1930. doi: 10.1038/oby.2006.224 
Vollaard, N. B. J., Shearman, J. P., and Cooper, C. E. (2005). Exerciseinduced oxidative stress:myths, realities and physiological relevance. Sports Med. 35, 1045-1062. doi: 10.2165/00007256-20053512000004

Werner, C. M., Hecksteden, A., Morsch, A., Zundler, J., Wegmann, M., Kratzsch, J., et al. (2018). Differential effects of endurance, interval, and resistance training on telomerase activity and telomere length in a randomized, controlled study. Eur. Heart J. 40, 34-46. doi: 10.1093/eurheartj/ ehy585

Xie, K., and Huang, S. (2003). Regulation of cancer metastasis by stress pathways. Clin. Exp. Metastasis. 20, 31-43.
Conflict of Interest Statement: The authors declare that the research was conducted in the absence of any commercial or financial relationships that could be construed as a potential conflict of interest.

Copyright (c) 2019 Gagnon, Dorman, Ritchie, Mutt, Stenbäck, Walkowiak and Herzig. This is an open-access article distributed under the terms of the Creative Commons Attribution License (CC BY). The use, distribution or reproduction in other forums is permitted, provided the original author(s) and the copyright owner(s) are credited and that the original publication in this journal is cited, in accordance with accepted academic practice. No use, distribution or reproduction is permitted which does not comply with these terms. 